- Original Article

\title{
Cancer Survivors with Low Hand Grip Strength Have Decreased Quality of Life Compared with Healthy Controls: The Korea National Health and Nutrition Examination Survey 2014-2017
}

\author{
Hyunji Kim, Sunmi Yoo*, Hyoeun Kim, Seung Guk Park, Minkyu Son \\ Department of Family Medicine, Inje University Haeundae Paik Hospital, Busan, Korea
}

\begin{abstract}
Background: Cancer survivors experience decreased physical function and reduced muscle strength, which leads to lower quality of life (QOL). The hand grip strength (HGS) can be a predictor of poor health-related QOL as a parameter of sarcopenia. The purpose of this study was to investigate the relationship between low HGS and QOL in cancer survivors and healthy controls.

Methods: We analyzed 392 cancer survivors and 1,176 healthy controls from the Korea National Health and Nutrition Examination Survey, 2014-2017. We defined low HGS as 2 standard deviation values for healthy young Korean adults from a previous study. QOL was evaluated using the European Quality of Life Scale-Five Dimensions. A complex sample logistic regression model was used to assess the relationship between each dimension of low HGS and QOL.

Results: The odds ratios (ORs) for decreased QOL were significantly higher in male cancer survivors with low HGS on self-care (OR, 8.51; 95\% confidence interval [CI], 1.69-42.83) and usual activities (OR, 6.63; 95\% CI, 1.22-36.03). The ORs for problems in mobility (OR, 5.87; 95\% CI, 2.04-16.91), usual activities (OR, 14.46; 95\% CI, 3.84-54.44), pain/discomfort (OR, 4.90; 95\% CI, 2.00-12.01), and anxiety/depression (OR, 6.43; 95\% CI, 2.16-19.12) were significantly high in female cancer survivors with low HGS. However, healthy controls showed no significant relationship between low HGS and QOL.

Conclusion: For cancer survivors, low HGS was associated with poor QOL in some domains. Strategies to increase muscle strength must be considered to improve the QOL of cancer survivors.
\end{abstract}

Keywords: Cancer Survivors; Hand Strength; Quality of Life; Sarcopenia

Received: March 30, 2020, Revised: June 12, 2020, Accepted: June 25, 2020

${ }^{*}$ Corresponding Author: Sunmi Yoo https://orcid.org/0000-0001-7407-8423

Tel: +82-51-797-3220, Fax: +82-51-797-3229, E-mail: syoo@paik.ac.kr 


\section{INTRODUCTION}

The number of cancer survivors has been steadily increasing due to the early detection of cancer, improvement in cancer treatment, and aging of the population. As the survival of cancer survivors is increasing, their quality of life (QOL) is becoming more important. Previous studies have shown that cancer survivors have a lower QOL than healthy subjects. ${ }^{1)}$ Factors that affect the QOL of cancer survivors include physical functioning, social functioning, emotional state, treatment and complications of cancer, types of cancer, and accompanying diseases. ${ }^{1-4)}$ Some cancer survivors have poor physical function, which is positively correlated with muscle strength. ${ }^{5)}$ Cancer is one of the causes of secondary sarcopenia, ${ }^{6}{ }^{6}$ which increases the likelihood of adverse outcomes, including falls, fractures, physical disability, and mortality, which can contribute to a decreased QOL. ${ }^{6,7)}$ The European Working Group on Sarcopenia in Older People recommends hand grip strength (HGS) measurements as a means of assessing low muscle strength, which can be a predictor of poor health-related QOL (HRQOL) as a parameter of sarcopenia. ${ }^{6)}$ HGS measurement is a simple and non-invasive method to assess muscle strength.

Low HGS and sarcopenia were associated with reduced HR-QOL in a cross-sectional study of community-dwelling older people. ${ }^{8)}$ Another study assessed the relationship between HGS and QOL in Korean men and women over the age of 19 and found that people with low HGS had poor QOL. ${ }^{9)}$ Studies have been conducted on the relationship between chronic diseases (diabetes, cardiovascular disease, chronic obstructive pulmonary disease, liver disease, etc.) and HGS or depression and HGS in Korean populations. ${ }^{10-14)}$ However, it is still controversial whether the HGS of cancer survivors is lower than that of healthy people since the relationship between HGS and QOL has rarely been evaluated for cancer survivors. A recent study found no difference between the HGS of cancer survivors and healthy controls, but there was an association between low HGS and poor QOL; as a limitation, the study had a small sample size. ${ }^{5)}$ Furthermore, research on the impact of HGS on HR-QOL in cancer survivors and healthy controls is still lacking.

Therefore, we used the data from the Korea National Health and Nutrition Examination Survey (KNHNES) to identify differences in HGS between cancer survivors and healthy controls. We also identified whether low HGS was associated with a decrease in QOL. We hypothesized that cancer survivors would have lower HGS than healthy controls, and low HGS would be related to poor QOL in both groups.

\section{METHODS}

\section{Study Participants}

This study was based on data collected from the KNHANES conducted from 2014 to 2017. It is a nationwide representative cross-sectional survey conducted by the Korea Centers for Disease Control and Prevention (KCDC, now Korea Disease Control and Prevention Agency). The participants of the KNHANES were selected using multistage stratified cluster sampling based on sex, age, and geographic area.
Among 24,821 adults aged $\geq 19$ years who participated in the NHANES, cancer survivors and healthy controls without a history of cancer were selected. We excluded those with diseases that could possibly influence HGS, such as hypertension, diabetes mellitus, stroke, angina, myocardial infarction, rheumatoid arthritis, osteoarthritis, or depression $(n=11,165)$. We also excluded those who did not have data for HGS, nutrition, and European Quality of Life Scale-Five Dimensions (EQ-5D) $(n=531)$. A total of 10,634 individuals were included in the study. Participants were then classified as cancer survivors if they had ever been diagnosed with cancer by a physician. The types of cancer were classified as stomach, liver, colon, lung, breast, cervical, and thyroid cancer. Patients with benign tumors, skin cancers, carcinoma in situ, or cancers of unknown origin were excluded from the cancer survivor group. As age and sex affected the incidence of cancer and HGS, we performed case-control matching by randomly assigning cancer survivors and healthy controls at a ratio of 1:3 by age and sex. Finally, a total of 392 individuals were included in the cancer survivor group, and 1,176 healthy controls were selected from the population without cancer $(n=10,242)$ (Figure 1). All participants provided informed consent, and this study was approved by the KCDC Institutional Review Board (2013-12EXP-03-5C, 2015-01-02-6C). For the 2016-2017 KNHANES data, Institutional Review Board approval was not necessary because the study was carried out for public welfare.

\section{Data Collection}

The KNHANES consists of a health interview survey, health examination, and nutrition survey. The participants were asked to provide data on sociodemographic factors, including age, sex, household income, education, and number of family members. Based on the national median household income, household income was categorized into quartiles: lowest ( $<25$ th percentile), medium-lowest $(<49$ th percentile), medium-highest (50th-74th percentile), and highest ( $\geq 75$ th per-

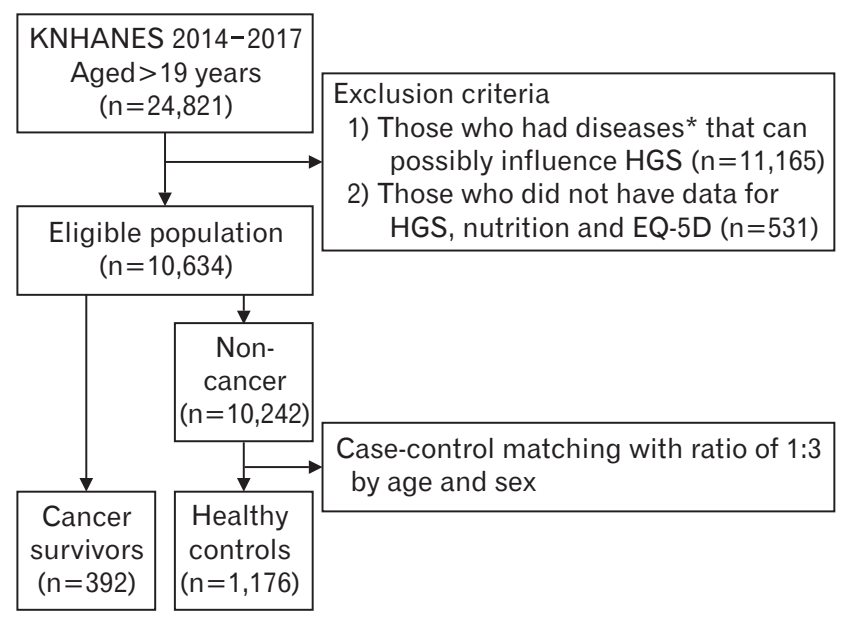

Figure 1. Flowchart of participants throughout the study. KNHANES, Korea National Health and Nutrition Examination Survey; HGS, hand grip strength; EQ-5D, European Quality of Life Scale-Five Dimensions. *Hypertension, diabetes mellitus, stroke, myocardial infarction, angina, rheumatoid arthritis, osteoarthritis, and depression. 
centile). Education level was classified as high school or university graduate. The number of family members living with the patients were recorded.

Lifestyle factors, including physical activity, alcohol consumption, and smoking status were collected. Physical activity was evaluated using the Korean version of the Global Physical Activity Questionnaire. Sufficient aerobic physical activity was defined as performing more than 2.5 hours of moderate-intensity activity per week or more than 1.25 hours of high-intensity activity per week or mixing moderate-and high-intensity physical activity ( 1 minute of high-intensity activity is equivalent to 2 minutes of moderate-intensity activity). Sufficient resistance exercise was defined as performing muscle-strengthening exercises for 2 days or more per week for the past 1 week. According to the Korean Ministry of Health and Welfare, risk drinking was defined as drinking more than 7 drinks for men and more than 5 drinks for women at least once a month during the past year. Smoking status was classified as non-smokers, who smoked less than five cigarettes in their lifetime, ex-smokers, who smoked more than 5 cigarettes in their lifetime, and current smokers who currently smoke.

Height was measured using a stadiometer in a standing straight position with no shoes. Body weight was measured using a weighing scale with light clothing and no shoes. Body mass index (BMI) was calculated as the weight divided by the square of height $\left(\mathrm{kg} / \mathrm{m}^{2}\right)$. The nutrition survey was conducted through face-to-face interviews. Daily total energy (kcal) and protein (g) intake was estimated using 24-hour recall and a food frequency questionnaire by a well-trained nutritionist.

\section{Hand Grip Strength}

HGS was measured with a digital HGS dynamometer (T.K.K 5401; Takei Scientific Instruments Co. Ltd., Tokyo, Japan) that measures between $5.0 \mathrm{~kg}$ and $100.0 \mathrm{~kg}$ of force with a precision of $0.1 \mathrm{~kg}$. The mea-

Table 1. Characteristics of cancer survivors and healthy controls $(n=1,568)$

\begin{tabular}{|c|c|c|c|c|c|c|}
\hline \multirow[b]{2}{*}{ Characteristic } & \multicolumn{3}{|c|}{ Male $(n=512)$} & \multicolumn{3}{|c|}{ Female $(n=1,056)$} \\
\hline & $\begin{array}{l}\text { Cancer survivors } \\
\qquad(\mathrm{n}=128)\end{array}$ & $\begin{array}{l}\text { Healthy controls } \\
\qquad(n=384)\end{array}$ & P-value & $\begin{array}{l}\text { Cancer survivors } \\
\qquad(\mathrm{n}=264)\end{array}$ & $\begin{array}{l}\text { Healthy controls } \\
\quad(n=792)\end{array}$ & P-value \\
\hline Age (y) & $59.47 \pm 1.87$ & $58.97 \pm 0.88$ & 0.801 & $51.40 \pm 0.73$ & $50.42 \pm 0.46$ & 0.254 \\
\hline Household income & & & 0.489 & & & 0.460 \\
\hline Low & $19.4(3.8)$ & $21.5(2.3)$ & & $9.0(1.7)$ & $10.5(1.1)$ & \\
\hline Low-mid & $27.8(4.4)$ & $20.8(2.3)$ & & $23.7(2.9)$ & $25.3(1.9)$ & \\
\hline Mid-high & $24.0(4.9)$ & $29.9(2.9)$ & & $33.9(3.4)$ & $28.3(1.8)$ & \\
\hline High & $28.8(5.3)$ & $27.8(2.7)$ & & $33.4(3.6)$ & $35.9(2.2)$ & \\
\hline \multicolumn{7}{|l|}{ Education } \\
\hline$\leq$ High school graduate or less & $66.5(5.3)$ & $61.8(3.0)$ & 0.431 & $67.1(3.4)$ & $65.4(2.2)$ & 0.665 \\
\hline Live alone & $7.3(2.5)$ & $9.2(1.6)$ & 0.540 & $6.1(1.3)$ & $6.1(0.8)$ & 0.962 \\
\hline \multicolumn{7}{|l|}{ Cancer } \\
\hline Gastric cancer & $36.2(4.8)$ & & & $9.9(2.1)$ & & \\
\hline Liver cancer & $4.4(1.8)$ & & & $0.2(0.2)$ & & \\
\hline Colon cancer & $16.4(3.6)$ & & & $6.7(1.8)$ & & \\
\hline Breast cancer & - & & & $20.9(2.9)$ & & \\
\hline Cervical cancer & - & & & $19.4(2.8)$ & & \\
\hline Lung cancer & $7.9(3.1)$ & & & $1.3(0.8)$ & & \\
\hline Thyroid cancer & $6.0(2.4)$ & & & $31.3(3.2)$ & & \\
\hline Others & $35.6(5.1)$ & & & $14.0(2.4)$ & & \\
\hline Time since diagnosis $\leq 5 \mathrm{y}$ & $61.5(5.1)$ & & & $57.8(3.4)$ & & \\
\hline Aerobic physical activity & $49.8(5.3)$ & $51.0(3.0)$ & 0.843 & $54.1(3.9)$ & $46.6(2.1)$ & 0.089 \\
\hline Resistance exercise $\geq 2 \mathrm{~d} / \mathrm{wk}$ & $27.2(4.4)$ & $30.4(2.7)$ & 0.549 & $19.0(2.7)$ & $13.9(1.4)$ & 0.082 \\
\hline Body mass index $\left(\mathrm{kg} / \mathrm{m}^{2}\right)$ & $22.89 \pm 0.38$ & $23.55 \pm 0.19$ & 0.118 & $22.59 \pm 0.20$ & $22.77 \pm 0.13$ & 0.443 \\
\hline Total caloric intake (kcal/d) & $2,092.97 \pm 109.65$ & $2,255.56 \pm 82.50$ & 0.224 & $1,706.54 \pm 52.97$ & $1,771.44 \pm 30.69$ & 0.305 \\
\hline Total protein intake (g/d) & $79.36 \pm 6.45$ & $85.25 \pm 8.31$ & 0.572 & $60.09 \pm 2.02$ & $62.86 \pm 1.31$ & 0.253 \\
\hline At risk drinking* & $38.6(6.7)$ & $50.4(3.5)$ & 0.132 & $19.2(3.9)$ & $26.2(2.2)$ & 0.140 \\
\hline Smoking & & & 0.056 & & & 0.922 \\
\hline Current smoker & $17.1(4.1)$ & $30.3(2.9)$ & & $4.0(1.4)$ & $3.9(0.8)$ & \\
\hline Ex-smoker & $60.3(5.1)$ & $48.8(2.9)$ & & $4.0(1.3)$ & $4.6(0.9)$ & \\
\hline Non smoker & $22.6(4.6)$ & $20.8(2.3)$ & & $92.0(1.9)$ & $91.5(1.2)$ & \\
\hline Hand grip strength in dominant hand (kg) & $34.91 \pm 1.20$ & $36.76 \pm 0.66$ & 0.165 & $22.97 \pm 0.63$ & $23.31 \pm 0.31$ & 0.631 \\
\hline Subjects with low hand grip strength ${ }^{\dagger}(\%)$ & $17.2(3.6)$ & $16.0(2.0)$ & 0.749 & $12.8(2.6)$ & $11.2(1.3)$ & 0.569 \\
\hline
\end{tabular}

Values are presented as estimated mean \pm SE or estimated \% (SE). P-values were for the complex samples cross tabulation for categorical variables and the complex samples general linear model for continuous variables. A P-value of less 0.05 was considered as statistically significant.

SE, standard error.

*Risk drinking was defined as drinking more than 7 drinks for men and more than 5 drinks for women at least once a month during the past year. ${ }^{\dagger}$ The cut off values were $28.9 \mathrm{~kg}$ in men and $16.8 \mathrm{~kg}$ in women. 
surements were performed three times for each hand alternately, and the maximum grip strength of the dominant hand was used for the analysis.

We defined low HGS as 2 standard deviation values below the values for healthy young Korean adults from a previous study because the distribution of HGS varies according to many factors such as age, sex, and ethnicity. The cut-off values were $28.9 \mathrm{~kg}$ for men and $16.8 \mathrm{~kg}$ for women. ${ }^{15)}$

\section{European Quality of Life Scale-Five Dimensions}

The Korean version of the EQ-5D was used as an indicator of the HRQOL. The EQ-5D consists of five dimensions of health status: mobility, self-care, usual activities, pain/discomfort, and anxiety/depression. The mobility dimension evaluates an individual's walking ability, and the self-care dimension assesses the ability to wash or dress alone. The usual activity dimension evaluates the ability to perform work, study, housework, family, or leisure activities. The pain/discomfort and anxiety/depression dimensions evaluated the level of pain, discomfort, anxiety, or depression. The participants rated their level of severity for each dimension using a three-level scale. Those who responded that they were in the second level (having some problems) or third level (having severe/extreme problems) were considered to have problems because the number of respondents who rated their QOL as the third level was very small. In addition to the five dimensions of the QOL surveys, the EQ-5D index was used. The EQ-5D was conducted by the KCDC in 2007, and the index was calculated by weighting each dimension. When the EQ-5D index is 1.000, it indicates optimal health state, and the lower the EQ-5D, the poorer the HR-QOL. ${ }^{16)}$

\section{Statistical Analysis}

Data were expressed as the estimated mean \pm standard error or estimated percentage (standard error). We compared the demographic and clinical characteristics between cancer survivors and healthy controls using complex sample cross-tabulation for categorical variables and complex samples general linear model for continuous variables. Complex sample cross-tabulation was used to compare the HGS and QOL between the two groups. The complex samples general linear model was used to check the difference in EQ-5D index between the two groups. The complex samples logistic regression model was used to assess the relationship between low HGS and each dimension of QOL, including the interaction term of the HGS and cancer diagnosis to confirm the joint effect. The odds ratio (OR) and corresponding 95\% confidence intervals (CIs) for decreased QOL were obtained after adjusting for age, BMI, household income, time to diagnosis, resistance exercise, total caloric intake, and smoking status. Statistical analysis was performed using IBM SPSS ver. 25.0 (IBM Corp., Armonk, NY, USA). Statistical significance was set at $\mathrm{P}<0.05$.

\section{RESULTS}

\section{Basic Characteristics of the Participants}

The basic characteristics of the cancer survivors and healthy controls are presented in Table 1. No significant differences were found in household income, education, living with some, performing aerobic physical activity and resistance exercise, BMI, total caloric intake, total protein intake, at-risk drinking, and smoking between the cancer survivors and healthy controls, for both men and women. The mean dominant HGS values of cancer survivors and healthy controls were $34.91 \pm 1.20 \mathrm{~kg}$ and $36.76 \pm 0.66 \mathrm{~kg}$, respectively, for men; and $22.97 \pm 0.63$ $\mathrm{kg}$ and $23.31 \pm 0.31 \mathrm{~kg}$, respectively, for women, showing no significant difference.

The most common cancers were gastric cancer in men and thyroid cancer in women. In cases where multiple cancers were diagnosed in one individual, the time since diagnosis was assessed using the time of the most recent diagnosis. The percentage of survivors diagnosed with cancer within 5 years was $61.5 \%$ for men and $57.8 \%$ for women.

\section{Quality of Life of Cancer Survivors and Healthy Controls}

Table 2 shows the EQ-5D index for cancer survivors and healthy controls. The EQ-5D index was found to be significantly lower in male cancer survivors than in healthy controls. Self-care and usual activities were significantly worse in male cancer survivors than in male healthy controls. Usual activities and anxiety/depression were significantly worse in female cancer survivors than in female healthy controls. The

Table 2. Comparison of EQ-5D between cancer survivors and healthy controls

\begin{tabular}{|c|c|c|c|c|c|c|}
\hline \multirow[b]{2}{*}{$E Q-5 D$} & \multicolumn{3}{|c|}{ Male $(n=512)$} & \multicolumn{3}{|c|}{ Female $(n=1,056)$} \\
\hline & $\begin{array}{l}\text { Cancer survivors } \\
\quad(n=128)\end{array}$ & $\begin{array}{l}\text { Healthy controls } \\
\qquad(n=384)\end{array}$ & P-value & $\begin{array}{l}\text { Cancer survivors } \\
\quad(\mathrm{n}=264)\end{array}$ & $\begin{array}{l}\text { Healthy controls } \\
\quad(n=792)\end{array}$ & P-value \\
\hline Mobility: have problems ${ }^{\star}$ & $14.8(3.4)$ & $9.9(1.7)$ & 0.153 & $7.9(1.9)$ & $7.3(1.0)$ & 0.784 \\
\hline Self-care: have problems* & $5.7(2.3)$ & $1.5(0.6)$ & 0.011 & $2.2(0.9)$ & $1.7(0.5)$ & 0.611 \\
\hline Usual activities: have problems* & $11.6(3.6)$ & $4.8(1.2)$ & 0.023 & $7.5(1.9)$ & $2.9(0.6)$ & 0.005 \\
\hline Pain/discomfort: have problems ${ }^{\star}$ & $24.6(4.6)$ & $15.6(2.2)$ & 0.059 & $22.8(2.9)$ & $20.7(1.7)$ & 0.537 \\
\hline Anxiety/depression: have problems* & $8.3(2.9)$ & $8.1(1.8)$ & 0.952 & $14.3(2.9)$ & $7.6(1.0)$ & 0.011 \\
\hline EQ-5D index & $0.94 \pm 0.01$ & $0.96 \pm 0.05$ & 0.030 & $0.95 \pm 0.07$ & $0.96 \pm 0.03$ & 0.053 \\
\hline
\end{tabular}

Values are presented as estimated \% (SE) or estimated mean \pm SE.

EQ-5D, European Quality of Life Scale-Five Dimensions; SE, standard error.

*EQ-5D has a three-level scale for each dimension, and subjects who responded that they were in the second level (having some problems) or third level (having severe/ extreme problems) were considered as having problems. 
EQ-5D index was lower in female survivors than in healthy controls with marginal significance $(\mathrm{P}=0.053)$.

\section{Relationship between Low Hand Grip Strength and Quality of Life}

Table 3 presents the ORs and 95\% CIs of decreased QOL by HGS for men. In the crude analyses, the ORs for decreased QOL were significantly higher in cancer survivors with low HGS on mobility and selfcare of the EQ-5D. The ORs for decreased QOL were significantly higher in healthy controls with low HGS on mobility, self-care, usual activities, and pain/discomfort dimensions of the EQ-5D. After adjusting for age, BMI, household income, time to diagnosis, resistance exercise, total caloric intake, and smoking, the ORs for problems in selfcare (OR, 8.51; 95\% CI, 1.69-42.83) and problems with usual activities (OR, 6.63; 95\% CI, 1.22-36.03) were found to be significantly high in cancer survivors with low HGS. On the other hand, after adjusting for covariates, the QOL of healthy controls was not related to low HGS in any dimension. The joint effect of cancer diagnosis and HGS for each dimension of QOL was not significant in men (all P for interaction $>0.05)$.

Table 4 shows the ORs and 95\% CIs of poor QOL according to HGS for women. In the crude analyses, the ORs for decreased QOL were significantly higher in cancer survivors with low HGS in all dimensions. The ORs for problems in mobility (OR, 5.87; 95\% CI, 2.04-16.91), usual activities (OR, 14.46; 95\% CI, 3.84-54.44), pain/discomfort (OR, 4.90; 95\% CI, 2.00-12.01), and anxiety/depression (OR, 6.43; 95\% CI, 2.16-19.12) were still significantly higher after adjusting for age, BMI, household income, time to diagnosis, resistance exercise, total caloric intake, and smoking. The QOL of female healthy controls did not show an association with low HGS at any dimension after adjusting for covariates. For women, the joint effect between cancer diagnosis and HGS was significant for pain/discomfort (P for interaction=0.004) and anxiety/depression ( $\mathrm{P}$ for interaction $=0.024)$, and marginally significant for mobility ( $\mathrm{P}$ for interaction=0.079) and usual activities ( $\mathrm{P}$ for interaction $=0.061)$.

\section{DISCUSSION}

This nationwide Korean study confirmed that low HGS was significantly associated with poor QOL in both male and female cancer survivors. Our results also demonstrated that there was no difference in the mean HGS between cancer survivors and healthy controls, and no significant relationship was found between HGS and QOL in healthy controls.

Previous studies have compared muscle strength between cancer survivors and the general population, and the results have been inconsistent. ${ }^{5,17-19)}$ Our research shows that the HGS of cancer survivors was not different from that of healthy controls, as in some prior studies. ${ }^{5,19)}$ This implies that the muscle strength of cancer survivors is similar to that of healthy subjects if their general health is in good condition. ${ }^{5)}$

We found that cancer survivors had problems with QOL in various domains compared to healthy controls. Pain/discomfort was most frequently problematic area (response, "have problems") for cancer survivors, as in previous studies. ${ }^{20,21)}$ Both male and female cancer survivors had problems with usual activity compared to healthy controls. Cancer-related symptoms such as anxiety, stress, depression, fatigue, or pain, whether resulting from the disease itself or the adverse effects of its treatment, are related to physical activity, which may affect the

Table 3. ORs and 95\% Cls for decreased QOL in male cancer survivors and healthy controls with low HGS*

\begin{tabular}{|c|c|c|c|c|c|c|c|c|c|}
\hline \multirow{3}{*}{$E Q-5 D$} & \multicolumn{4}{|c|}{ Cancer survivors $(n=128)$} & \multicolumn{4}{|c|}{ Healthy controls $(n=384)$} & \multirow{3}{*}{$\begin{array}{c}\text { P for } \\
\text { interaction }\end{array}$} \\
\hline & \multicolumn{2}{|l|}{ Crude } & \multicolumn{2}{|c|}{ Multi-adjusted $^{\dagger}$} & \multicolumn{2}{|l|}{ Crude } & \multicolumn{2}{|c|}{ Multi-adjusted $^{\dagger}$} & \\
\hline & OR $(95 \% \mathrm{Cl})$ & $P$-value & OR $(95 \% \mathrm{Cl})$ & P-value & OR $(95 \% \mathrm{Cl})$ & P-value & OR $(95 \% \mathrm{Cl})$ & P-value & \\
\hline \multicolumn{10}{|c|}{ Problem in mobility ${ }^{\S}$} \\
\hline Normal HGS & 1.00 & & 1.00 & & 1.00 & & 1.00 & & \\
\hline Low HGS* & $4.59(1.41-14.98)$ & 0.012 & $2.09(0.61-7.16)$ & 0.237 & 4.65 (2.19-9.87) & $<0.001$ & $1.78(0.65-4.87)$ & 0.260 & 0.905 \\
\hline \multicolumn{10}{|c|}{ Problem in self-care ${ }^{\S}$} \\
\hline Normal HGS & 1.00 & & 1.00 & & 1.00 & & 1.00 & & \\
\hline Low HGS* & $7.07(1.30-38.59)$ & 0.024 & $8.51(1.69-42.83)$ & 0.010 & $13.53(1.56-117.14)$ & 0.018 & $2.25(0.29-17.55)$ & 0.438 & 0.643 \\
\hline \multicolumn{10}{|c|}{ Problem in usual activities ${ }^{\S}$} \\
\hline Normal HGS & 1.00 & & 1.00 & & 1.00 & & 1.00 & & \\
\hline Low HGS* & $3.70(0.91-15.11)$ & 0.068 & $6.63(1.22-36.03)$ & 0.029 & $5.89(2.11-16.45)$ & 0.001 & $2.47(0.56-10.87)$ & 0.230 & 0.918 \\
\hline \multicolumn{10}{|l|}{ Pain/discomfort ${ }^{\S}$} \\
\hline Normal HGS & 1.00 & & 1.00 & & 1.00 & & 1.00 & & \\
\hline Low HGS* & $1.63(0.51-5.17)$ & 0.408 & $2.67(0.90-8.00)$ & 0.077 & $2.29(1.14-4.62)$ & 0.020 & $1.33(0.54-3.30)$ & 0.532 & 0.923 \\
\hline \multicolumn{10}{|l|}{ Anxiety/depression $\$$} \\
\hline Normal HGS & 1.00 & & 1.00 & & 1.00 & & $1.00 \mathrm{C}$ & & \\
\hline Low HGS* & $3.88(0.88-17.17)$ & 0.074 & $2.73(0.44-17.14)$ & 0.280 & $1.64(0.62-4.30)$ & 0.318 & $0.98(0.37-2.60)$ & 0.962 & 0.482 \\
\hline
\end{tabular}

OR, odds ratio; $\mathrm{Cl}$, confidence interval; QOL, quality of life; HGS, hand grip strength; EQ-5D, European Quality of Life Scale-Five Dimensions.

${ }^{\star}$ The cut-off value for low HGS was $28.9 \mathrm{~kg}$ in men. There were 102 men with normal HGS and 26 men with low HGS among cancer survivors. In the healthy controls, there were 303 men with normal HGS and 81 men with low HGS. ${ }^{\dagger}$ Adjusted for age, body mass index, household income, time to diagnosis (excluded from healthy control group), resistance exercise, total caloric intake, and smoking. ${ }^{\ddagger}$ Interaction between cancer diagnosis and hand grip strength. ${ }^{\circledR} E Q-5 D$ has a three-level scale for each dimension, and subjects who responded that they were in the second level (having some problems) or third level (having severe/extreme problems) were considered as having problems. 
Table 4. ORs and 95\% Cls for decreased QOL in female cancer survivors and healthy controls with low HGS*

\begin{tabular}{|c|c|c|c|c|c|c|c|c|c|}
\hline \multirow{3}{*}{$E Q-5 D$} & \multicolumn{4}{|c|}{ Cancer survivors $(n=264)$} & \multicolumn{4}{|c|}{ Healthy controls ( $\mathrm{n=792)}$} & \multirow{3}{*}{$\begin{array}{c}\text { P for } \\
\text { interaction }\end{array}$} \\
\hline & \multicolumn{2}{|l|}{ Crude } & \multicolumn{2}{|l|}{ Multi-adjusted $^{\dagger}$} & \multicolumn{2}{|l|}{ Crude } & \multicolumn{2}{|c|}{ Multi-adjusted $^{\dagger}$} & \\
\hline & OR (95\% Cl) & P-value & $\mathrm{OR}(95 \% \mathrm{Cl})$ & P-value & OR $(95 \% \mathrm{Cl})$ & P-value & OR $(95 \% \mathrm{Cl})$ & P-value & \\
\hline \multicolumn{10}{|c|}{ Problem in mobility $\S^{\S}$} \\
\hline Normal HGS & 1.00 & & 1.00 & & 1.00 & & 1.00 & & \\
\hline Low HGS* & $7.00(2.21-22.20)$ & 0.001 & $5.87(2.04-16.91)$ & 0.001 & $2.80(1.45-5.44)$ & 0.002 & $1.06(0.50-2.22)$ & 0.888 & 0.079 \\
\hline \multicolumn{10}{|c|}{ Problem in self-care ${ }^{\S}$} \\
\hline Normal HGS & 1.00 & & 1.00 & & 1.00 & & 1.00 & & \\
\hline Low HGS* & 7.01 (1.24-39.52) & 0.028 & $8.85(0.49-158.37)$ & 0.138 & $4.61(1.50-14.20)$ & 0.008 & $1.49(0.26-8.46)$ & 0.656 & 0.438 \\
\hline \multicolumn{10}{|c|}{ Problem in usual activities ${ }^{\S}$} \\
\hline Normal HGS & 1.00 & & 1.00 & & 1.00 & & 1.00 & & \\
\hline Low HGS* & $11.44(3.43-38.18)$ & $<0.001$ & $14.46(3.84-54.44)$ & $<0.001$ & $4.08(1.64-10.15)$ & 0.003 & $2.40(0.80-7.19)$ & 0.117 & 0.061 \\
\hline \multicolumn{10}{|l|}{ Pain/discomfort ${ }^{\S}$} \\
\hline Normal HGS & 1.00 & & 1.00 & & 1.00 & & 1.00 & & \\
\hline Low HGS* & $4.53(1.79-11.46)$ & 0.001 & $4.90(2.00-12.01)$ & 0.001 & $1.30(0.75-2.25)$ & 0.354 & $1.20(0.64-2.24)$ & 0.571 & 0.004 \\
\hline \multicolumn{10}{|c|}{ Anxiety/depression $\S$} \\
\hline Normal HGS & 1.00 & & 1.00 & & 1.00 & & 1.00 & & \\
\hline Low HGS* & $6.87(2.43-19.37)$ & $<0.001$ & $6.43(2.16-19.12)$ & 0.001 & $1.38(0.68-2.81)$ & 0.371 & $1.00(0.45-2.23)$ & 0.998 & 0.024 \\
\hline
\end{tabular}

$\mathrm{OR}$, odds ratio; $\mathrm{Cl}$, confidence interval; QOL, quality of life; HGS, hand grip strength; EQ-5D, European Quality of Life Scale-Five Dimensions.

${ }^{*}$ The cut-off value for low HGS was $16.8 \mathrm{~kg}$ women. There were 234 women with normal HGS and 30 women with low HGS among cancer survivors. In the healthy controls, there were 693 women with normal HGS and 99 women with low HGS. ${ }^{\dagger}$ Adjusted for age, BMl, household income, time to diagnosis (excluded from healthy control group), resistance exercise, total caloric intake, and smoking. ${ }^{\ddagger}$ Interaction between cancer diagnosis and hand grip strength. ${ }^{8} \mathrm{EQ}-5 \mathrm{D}$ has a three-level scale for each dimension, and subjects who responded that they were in the second level (having some problems) or third level (having severe/extreme problems) were considered as having problems.

usual activities. ${ }^{22)}$ Unlike men, female cancer survivors experienced anxiety/depression more than the healthy controls in this study. Breast cancer survivors had a lower QOL than the general population in overall HR-QOL, where pain/discomfort and anxiety/depression lasted for years. ${ }^{23,24)}$ Female cancer survivors seem to find it more difficult to recover their QOL in the emotional domain.

In previous studies of a small number of cancer survivors, low HGS was associated with poor QOL. ${ }^{5,21,25)}$ A recent study using the KNHANES showed that weak HGS was associated with impaired HR-QOL. However, it was unclear whether the results were specific to cancer survivors or applicable to the general population since the study included only cancer survivors. ${ }^{21)}$ We directly compared cancer survivors with healthy controls by case-control matching and checked the interaction between cancer diagnosis and HGS to determine whether low HGS has a greater impact on QOL in cancer survivors than in healthy controls. As a result, it was confirmed that cancer diagnosis affects the relationship between HGS and QOL in women. Our results suggest that low muscle strength can be a risk factor affecting the QOL of cancer survivors compared to healthy controls. There was no significant difference in the HGS between cancer survivors and healthy controls, but the scores for the physical components of QOL, such as mobility and usual activities, were lower in cancer survivors than in healthy controls. HGS is closely correlated with leg muscle strength and provides a valid marker of limb muscle strength. ${ }^{26)}$ Previous studies have shown that high knee muscle strength is associated with an increased QOL. ${ }^{5}$ Thus, the relationship between low HGS and poor QOL seems to be stronger in vulnerable populations such as cancer survivors and patients with chronic diseases than in healthy people. ${ }^{10-13)}$ Female cancer survivors with low HGS, who had poor QOL in all domains except self-care in our study, can also be considered vulnerable. A prior Korean cross-sectional study reported that lower HGS was associated with poor QOL in healthy adults. ${ }^{9)}$ However, the study included people with arthritis and depression, excluded cancer survivors, and adopted quartiles to define low HGS cut-off values. Further research is needed to confirm which interventions are effective in improving the QOL of vulnerable populations with weak muscle strength. Exercise should be an option for several interventions, as resistance and aerobic exercise improve the QOL for

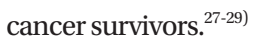

Our results should be interpreted in the context of several limitations. The cross-sectional nature of the study did not prove a causal relationship between HGS and QOL. Contrary to our hypothesis, cancer survivors who already have a high QOL can have sufficient muscle strength to maintain their physical functions and live independently. Second, it is unclear whether the results of this study are due to cancer itself, the effects of treatment, or the characteristics of survivors. We did not have detailed medical information regarding the cancer stage and therapeutic methods. Future studies with a prospective design comparing HGS and QOL before and after treatment may provide an answer. More than half of cancer survivors were within 5 years of diagnosis, but it was not possible to distinguish whether they were under active treatment or after treatment was completed at the time of the investigation. Finally, we did not analyze muscle mass or physical performance to evaluate sarcopenia in cancer survivors.

Nevertheless, our results are meaningful because this was a largescale population-based study, and we conducted a case-control matching analysis with case-control ratios of 1:3 by age and sex. We applied strict inclusion criteria to exclude confounding variables that could affect muscle strength and QOL. This study defined low HGS by 
using the cut-off values identified in a previous study of healthy young Korean adults instead of using arbitrarily determined critera. ${ }^{15)}$

In conclusion, low HGS was closely related to poor QOL among Korean cancer survivors. In particular, female cancer survivors with low HGS were at a risk of poor QOL in most domains. Muscle strength evaluation with HGS can be a useful tool for identifying populations vulnerable to poor QOL. Future research is needed to design various interventions to increase muscle strength and improve the QOL of cancer survivors.

\section{CONFLICT OF INTEREST}

No potential conflict of interest relevant to this article was reported.

\section{ORCID}

Hyunji Kim: https://orcid.org/0000-0003-1965-9406

Sunmi Yoo: https://orcid.org/0000-0001-7407-8423

Hyoeun Kim: https://orcid.org/0000-0001-7078-7599

Seung Guk Park: https://orcid.org/0000-0002-2986-3729

Minkyu Son: https://orcid.org/0000-0001-8817-6112

\section{REFERENCES}

1. Annunziata MA, Muzzatti B, Flaiban C, Gipponi K, Carnaghi C, Tralongo $\mathrm{P}$, et al. Long-term quality of life profile in oncology: a comparison between cancer survivors and the general population. Support Care Cancer 2018;26:651-6.

2. Miller KD, Triano LR. Medical issues in cancer survivors: a review. Cancer J 2008;14:375-87.

3. Gegechkori N, Haines L, Lin JJ. Long-term and latent side effects of specific cancer types. Med Clin North Am 2017;101:1053-73.

4. Vissers PA, Thong MS, Pouwer F, Zanders MM, Coebergh JW, van de Poll-Franse LV. The impact of comorbidity on Health-Related Quality of Life among cancer survivors: analyses of data from the PROFILES registry. J Cancer Surviv 2013;7:602-13.

5. Morishita S, Tsubaki A, Fu JB, Mitobe Y, Onishi H, Tsuji T. Cancer survivors exhibit a different relationship between muscle strength and health-related quality of life/fatigue compared to healthy subjects. Eur J Cancer Care (Engl) 2018;27:e12856.

6. Cruz-Jentoft AJ, Bahat G, Bauer J, Boirie Y, Bruyere O, Cederholm T, et al. Sarcopenia: revised European consensus on definition and diagnosis. Age Ageing 2019;48:16-31.

7. Beaudart C, Biver E, Reginster JY, Rizzoli R, Rolland Y, Bautmans I, et al. Validation of the SarQoL(R), a specific health-related quality of life questionnaire for sarcopenia. J Cachexia Sarcopenia Muscle 2017;8: 238-44.

8. Marques LP, Confortin SC, Ono LM, Barbosa AR, d'Orsi E. Quality of life associated with handgrip strength and sarcopenia: EpiFloripa Aging Study. Arch Gerontol Geriatr 2019;81:234-9.

9. Kang SY, Lim J, Park HS. Relationship between low handgrip strength and quality of life in Korean men and women. Qual Life Res 2018;27: 2571-80.
10. Jang M, Kim H, Oh B. Comparison of hand-grip strength between normal Korean adults and those with type 2 diabetes: 2014-2015 Korea National Health and Nutrition Examination Survey. Korean J Fam Pract 2018;8:654-61.

11. Lee MR, Jung SM, Kim HS, Kim YB. Association of muscle strength with cardiovascular risk in Korean adults: findings from the Korea National Health and Nutrition Examination Survey (KNHANES) VI to VII (2014-2016). Medicine (Baltimore) 2018;97:e13240.

12. Jeong M, Kang HK, Song P, Park HK, Jung H, Lee SS, et al. Hand grip strength in patients with chronic obstructive pulmonary disease. Int J Chron Obstruct Pulmon Dis 2017;12:2385-90.

13. Kim BJ, Ahn SH, Lee SH, Hong S, Hamrick MW, Isales CM, et al. Lower hand grip strength in older adults with non-alcoholic fatty liver disease: a nationwide population-based study. Aging (Albany NY) 2019; 11:4547-60.

14. Lee MR, Jung SM, Bang H, Kim HS, Kim YB. The association between muscular strength and depression in Korean adults: a cross-sectional analysis of the sixth Korea National Health and Nutrition Examination Survey (KNHANES VI) 2014. BMC Public Health 2018;18:1123.

15. Kim CR, Jeon YJ, Kim MC, Jeong T, Koo WR. Reference values for hand grip strength in the South Korean population. PLoS One 2018;13: e0195485.

16. Nam HS, Kim KY, Kweon SS, Koh KW, Kind P. Estimation report on quality weights for EQ-5D. Cheongju: Korea Disease Control and Prevention Agency; 2007.

17. Yee J, Davis GM, Beith JM, Wilcken N, Currow D, Emery J, et al. Physical activity and fitness in women with metastatic breast cancer. J Cancer Surviv 2014;8:647-56.

18. Fong SS, Ng SS, Luk WS, Chung JW, Chung LM, Tsang WW, et al. Shoulder mobility, muscular strength, and quality of life in breast cancer survivors with and without Tai Chi Qigong training. Evid Based Complement Alternat Med 2013;2013:787169.

19. Gannon JA, Guinan EM, Doyle SL, Beddy P, Reynolds JV, Hussey J. Reduced fitness and physical functioning are long-term sequelae after curative treatment for esophageal cancer: a matched control study. Dis Esophagus 2017;30:1-7.

20. Oh MG, Han MA, Park CY, Park SG, Chung CH. Health-related quality of life among cancer survivors in Korea: the Korea National Health and Nutrition Examination Survey. Jpn J Clin Oncol 2014;44:153-8.

21. Paek J, Choi YJ. Association between hand grip strength and impaired health-related quality of life in Korean cancer survivors: a cross-sectional study. BMJ Open 2019;9:e030938.

22. Albrecht TA, Taylor AG. Physical activity in patients with advancedstage cancer: a systematic review of the literature. Clin J Oncol Nurs 2012;16:293-300.

23. Rim CH, Ahn SJ, Kim JH, Yoon WS, Chun M, Yang DS, et al. An assessment of quality of life for early phase after adjuvant radiotherapy in breast cancer survivors: a Korean multicenter survey (KROG 14-09). Health Qual Life Outcomes 2017;15:96.

24. Yu J, Son WS, Lee SB, Chung IY, Son BH, Ahn SH, et al. Uneven recovery patterns of compromised health-related quality of life (EQ-5D-3 L) domains for breast Cancer survivors: a comparative study. Health Qual Life Outcomes 2018;16:143.

25. Takayama K, Atagi S, Imamura F, Tanaka H, Minato K, Harada T, et al. Quality of life and survival survey of cancer cachexia in advanced non- 
small cell lung cancer patients: Japan nutrition and QOL survey in patients with advanced non-small cell lung cancer study. Support Care Cancer 2016;24:3473-80.

26. Bohannon RW, Magasi SR, Bubela DJ, Wang YC, Gershon RC. Grip and knee extension muscle strength reflect a common construct among adults. Muscle Nerve 2012;46:555-8.

27. Kalter J, Kampshoff CS, Chinapaw MJ, van Mechelen W, GalindoGarre F, Schep G, et al. Mediators of exercise effects on HRQoL in cancer survivors after chemotherapy. Med Sci Sports Exerc 2016;48:1859-
65.

28. Vardar Yagli N, Sener G, Arikan H, Saglam M, Inal Ince D, Savci S, et al. Do yoga and aerobic exercise training have impact on functional capacity, fatigue, peripheral muscle strength, and quality of life in breast cancer survivors? Integr Cancer Ther 2015;14:125-32.

29. Smith TM, Broomhall CN, Crecelius AR. Physical and psychological effects of a 12-session cancer rehabilitation exercise program. Clin J Oncol Nurs 2016;20:653-9. 